\title{
Intersection Control Model for Connected Vehicles
}

\author{
Andrei Klimovich \\ Department of Intelligent Information Technologies \\ Brest State Technical University \\ Brest, Belarus
}

\author{
Vasilii Shuts \\ Department of Intelligent Information Technologies \\ Brest State Technical University \\ Brest, Belarus
}

\begin{abstract}
In the modern world, intelligent transportation systems, such as driverless and connected vehicles, are rapidly developing. Introduction of these technologies to the mass market will lead to the new generation of efficient and safe traffic control systems. We present a traffic control model for isolated intersection, which uses advantages of autonomy and connectivity of vehicles to achieve better throughput. Model is based on usage V2I-communication between vehicles and intersection controller to improve efficiency due to more complete traffic flow information and possibility to give orders to separate vehicles.
\end{abstract}

Index Terms - traffic control, V2I-communication, autonomous vehicles, multiagent systems.

\section{INTRODUCTION}

Modern vehicular communication such as V2V (Vehicleto-Vehicle), or V2I (Vehicle-to-Infrastructure) are based on wireless communication standards: DSRC (Dedicated shortrange communications), WAVE (Wireless Access in Vehicular Environments), IEEE 802.11p [1]. One of the main application for these technologies is improvement of the traffic control algorithms to increase the capacity of transport networks [2]. The general principle of traffic organization at the intersection with the use of V2I-interaction is the installation of a special device-transmitter (RSU - road side unit) connected via a wired network to a server or a local traffic control center (Fig. 1). The transmitter has a certain range of action. Vehicles in this range are able to communicate with RSU and send their position, speed, route. Based on the collected information the system generates commands for individual cars or groups according to the control algorithm. As a result the system optimizes the order of passing the intersection and ensures efficient and safe use of its throughput. Improvement of control efficiency is achieved through more detailed information about traffic flows and the possibility to influence on them through the transmission of messages. Despite there are several proposed models for organizing the movement according to the described scheme [3, 4, 5], none of them can pretend to become a replacement for the classic traffic light regulation.

\section{INTERSECTION CONTROL MODEL}

The aim of the traffic control system is to solve an optimization problem minimizing the total time that cars spent on the intersection. Parameters of optimization problem are the duration and types of phases, where each phase is a set of

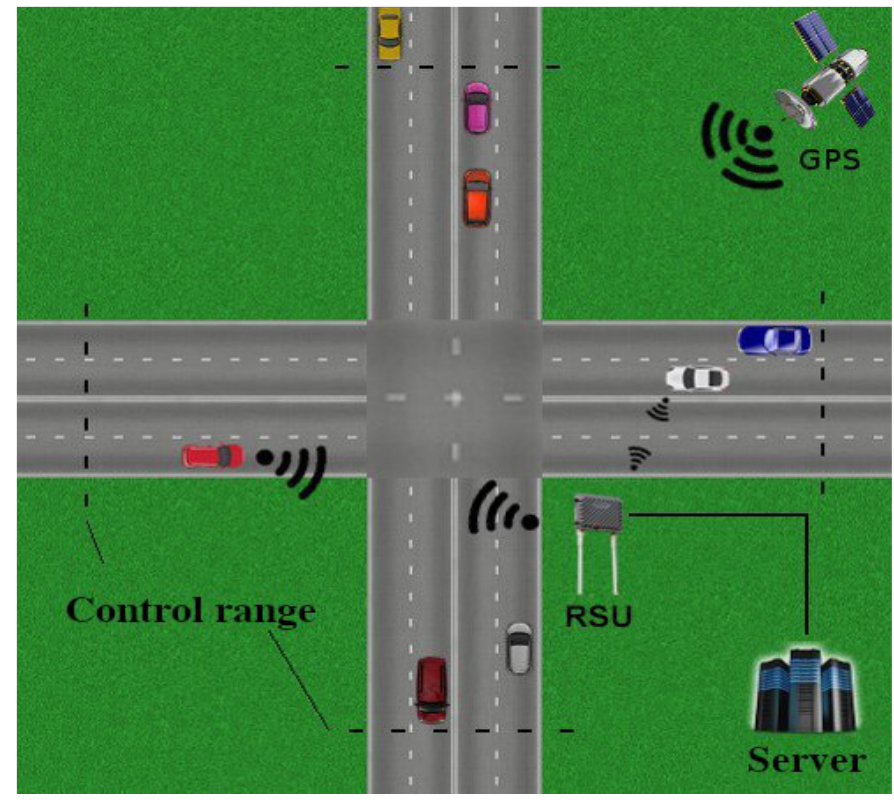

Figure 1. Intersection control scheme

permitted directions for movement. One of the main requirements is that the phase should not contain conflicting directions, i.e. trajectories of vehicles moving along these directions should not overlap. When planning phases, algorithm should take into account the number of cars moving along each road in a certain direction, the speed of these cars, the availability of maneuvering opportunities, and the change of lanes. After completing the planning, the system calculates for each vehicle the recommended arrival time to the intersection and sends them commands for acceleration, deceleration, lane change, etc. This process is repeated at regular intervals in order to process newly arrived cars and exclude cars that crossed the intersection. The number of different phases is small, for example, for the intersection of single-lane roads there are only 8 different optimal phases, they are shown in Fig. 2. In the case of multi-lane roads, the number of phases can be increased.

Let's describe the optimization problem we are solving. There are $\mathrm{N}$ cars at the time moment $\mathrm{t}_{0}$ within the system control range. For each car we know its route or direction $d_{i}$, speed $v_{i}$ and distance to the intersection $1_{i}$. Also it's important to consider arrival and departure time for each vehicle: $t_{i, a}$ and $t_{i, d}$. Then the whole time that vehicle spends on intersection can 


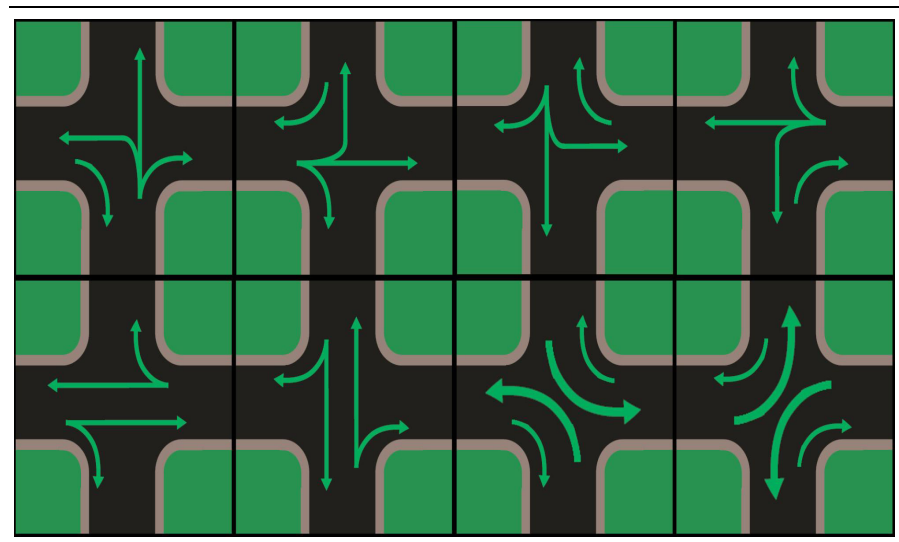

Figure 2. Intersection phases

be calculated as $T_{i}=t_{i, d}-t_{i, a}$. Thus, the optimization task is to minimize the average travel time for all cars as shown in (1):

$$
\left(\mathrm{T}_{1}+\ldots+\mathrm{T}_{\mathrm{N}}\right) / \mathrm{N} \rightarrow \min
$$

Estimated value of $\mathrm{T}_{\mathrm{i}}$ depends on the chosen phases, vehicle order, position and speed. To simplify model we suggest to take the duration of phases multiple of a certain short time interval, for example, $\Delta \mathrm{t}=10 \mathrm{~s}$. Now the task is reduced to choosing the phase for each time interval and determining the set of vehicles passing the intersection at these intervals. For this we propose to use the following algorithm:

1) Identify the cars that can pass the intersection at the end of the first time interval.

2) Choose the phase that allows to pass through the intersection to the largest number of cars.

3) If there are cars that could not cross the intersection in the current time interval, then go to the step 1, but for the next time interval. Otherwise, go to the step 4.

4) At this step number of the phase when vehicle should cross the intersection is known for all vehicles. Having this information system gives commands to the cars for acceleration or deceleration.

These steps are repeated in short intervals to process new incoming cars and to correct various deviations of the actual situation from the calculations. Let's describe the algorithm steps in more detail. First, cars on each road must be sorted in ascending distance to the intersection and processed in this order. At the first step for each car we determine the minimum time, through which the car reaches the intersection. We assume that the car accelerates to the maximum speed, and then moves uniformly. If we know maximal speed $\mathrm{v}_{\max }$ and maximal acceleration $\mathrm{a}_{\max }$, then acceleration time can be found from (2).

$$
\mathrm{t}_{\mathrm{ac}}=\left(\mathrm{v}_{\max }-\mathrm{v}_{\mathrm{i}}\right) / \mathrm{a}_{\max }
$$

And time to reach intersection for i-th car can be found from (3).

$$
\mathrm{t}=\mathrm{t}_{\mathrm{ac}}+\left(\mathrm{l}_{\mathrm{i}}-\mathrm{v}_{\mathrm{i}} \mathrm{t}_{\mathrm{ac}}-\mathrm{a}_{\max } \mathrm{t}_{\mathrm{ac}}{ }^{2} / 2\right) / \mathrm{v}_{\max }
$$

All cars that have the time $t$ smaller than the end time of the phase are involved in the phase determination in second step of the algorithm. On this step all possible phases are tested and the one which allows the maximal number of vehicles to pass the intersection is chosen. It is important to note that when determining the number of cars passing through the intersection, their order is taken into account. If phase forbids movement for some car, than all subsequent cars are blocked. The commands sent by controller at the fourth step determine the car movement until it reaches the intersection, they can be represented as a set of acceleration-duration pairs. Those cars that must pass through the intersection during the current phase should accelerate to the maximum allowed speed and then move uniformly. For other cars commands should be the following: slow down to a certain speed, move with it for a while and, finally, accelerate before the intersection to reduce time on intersection. Thus, the trajectory is divided into three sections: deceleration, uniform motion and acceleration. To determine these sections we should find their duration $\Delta \mathrm{t}_{1}, \Delta \mathrm{t}_{2}$ and $\Delta t_{3}$ and speed for second interval $v_{2}$. If we define acceptable deceleration $a_{d}$ and time when car should come to the intersection $\Delta t$, then unknown parameters of the trajectory can be found from system of equations (4), (5), (6) and (7):

$$
\begin{gathered}
\Delta \mathrm{t}_{1}=\left(\mathrm{v}_{2}-\mathrm{v}_{\mathrm{i}}\right) / \mathrm{a}_{\mathrm{d}} \\
\Delta \mathrm{t}_{3}=\left(\mathrm{v}_{\max }-\mathrm{v}_{2}\right) / \mathrm{a}_{\max } \\
\Delta \mathrm{t}=\Delta \mathrm{t}_{1}+\Delta \mathrm{t}_{2}+\Delta \mathrm{t}_{3}+\Delta \mathrm{t}_{4} \\
\mathrm{l}_{\mathrm{i}}=\mathrm{v}_{\mathrm{i}} \Delta \mathrm{t}_{1}-\mathrm{a}_{\mathrm{d}} \Delta \mathrm{t}_{1}{ }^{2} / 2+\mathrm{v}_{2} \Delta \mathrm{t}_{2}+\mathrm{v}_{2} \Delta \mathrm{t}_{3}+\mathrm{a}_{\max } \Delta \mathrm{t}_{3}{ }^{2} / 2
\end{gathered}
$$

The solution of the system completely determines the trajectory of the car. The computational complexity of the algorithm depends on the number of cars at the intersection $\mathrm{N}$, the number of different phases $M$ and the duration of the planning period (the number of time intervals for which phases are calculated $\mathrm{K})$. The final complexity is $\mathrm{O}(\mathrm{N} \log (\mathrm{N})+\mathrm{NMK})$ where the first term is responsible for sorting cars according to the distance to the intersection, and the second - for selecting the phases. As the values of $\mathrm{M}$ and $\mathrm{K}$ are small, even devices with low processing power can deal with it.

\section{CONCLUSION}

In this paper we described intersection control model for connected vehicles and proposed the algorithm that uses V2Icommunication to control trajectory of the vehicles and optimize throughput of the intersection. We considered a variant of the problem for a crossroads of single-lane roads. And in this case the model has significant limitations such as impossibility of changing the order of moving cars. This possibility arises even if there are at least two lanes, and this makes it even more efficient to manage the traffic flow. The model becomes complicated in this case, therefore it's necessary to monitor the computational complexity of the algorithms, and also to apply simulation modeling to determine model efficiency.

\section{REFERENCES}

[1] G.R. Hiertz, D. Denteneer, L. Stibor, "The IEEE 802.11 Universe," IEEE Communications Magazine, vol. 48(1), 2010, pp. 62-70.

[2] A. Perallos, U. Hernandez-Jayo, E. Onieva, "Intelligent transport systems: technologies and applications," John Wiley \& Sons, 2016.

[3] K. Dresner, P. Stone, "Multiagent Approach to Autonomous Intersection Management," Journal of Artificial Intelligence Research, vol. 31, 2008, pp. 591-656.

[4] Y. Fei, M. Dridi, A. El-Moudni, "New vehicle sequencing algorithms with vehicular infrastructure integration for an isolated intersection," Telecommunication Systems, 2012, pp. 325-337.

[5] Z. Li, L. Elefteriadou, S. Ranka, "Signal control optimization for automated vehicles at isolated signalized intersections," Transportation Research Part C 49, 2014, pp. 1-18. 